\title{
A Water-Quality Dynamic Monitoring System Based on Web-Server-Embedded Technology for Aquaculture
}

\author{
Dongxian $\mathrm{He}^{1}$, Daoliang $\mathrm{Li}^{2}$, Jie Bao ${ }^{3}, \mathrm{Hu}$ Juanxiu ${ }^{1}$, and Shaokun $\mathrm{Lu}^{1}$ \\ ${ }^{1}$ Key Lab. of Agriculture Engineering in Structure and Environment, \\ College of Water Conservation and Civil Engineering, China Agricultural University, \\ Beijing, P.R. China \\ ${ }^{2}$ College of Information and Electrical Engineering, China Agricultural University, \\ Beijing, P.R. China \\ ${ }^{3}$ College of Engineering, China Agricultural University, Beijing, P.R. China \\ he_dongxian@hotmail.com
}

\begin{abstract}
Water-quality monitoring technology has been taken a great attention in aquaculture because of increasing aquatic products and impact of food safety vicious incidents. In order to meet the technology requirements of water-quality monitoring in multi-parameter, dynamic, and networked monitoring, a waterquality dynamic monitoring system was developed to monitor multi-parameter water-quality variables such as water temperature, $\mathrm{pH}$, dissolved oxygen, electrical conductivity, oxidation reduction potential dynamically, and communicate with remote information servers in a high security. As result of a two-years testing experiment in a seawater aquiculture company, relative errors of each water-quality parameter measured by the instrument were less than 5\%, data lost percentage of remote communication was less than $3 \%$, and power energy consumption was less than $13 \mathrm{~W}$. Therefore, the water-quality dynamic monitoring system as a node infrastructure of wireless sensor networks can be used to achieve a distributed water-quality network monitoring system in a large-scale aquaculture farm, or to construct a distributed water-quality monitoring network system in multipoint of different cities for aquaculture farm and administration section.
\end{abstract}

Keywords: Dissolved oxygen, Electrical conductivity, Infrastructure, Oxidation reduction potential.

\section{Introduction}

Aquatic products play an important role in agricultural products. Farmed aquatic products reached the proportion of the total output of aquatic products $66.5 \%$ in China [1]. Aquaculture has becoming to an important industry in agriculture and the monitoring of water quality is an important issue for improving the aquatic production and quality. Effective water-quality monitoring systems are required to apply in aquaculture to measure and control water temperature, $\mathrm{pH}$, dissolved oxygen, and other important water quality parameters in abroad [2-6]. Water quality was regulated by 
physical, chemical and biological methods to organize the aquaculture production by utilizing hazard analysis and critical control point (HACCP) operating rules in Europe [7]. The computer-based process control technology proposed by United States for intensive aquaculture system can be used in marine fish's growth environment control [8]. Water-quality monitoring instruments and systems are trended to be smaller, more automatic and intelligent, and multi-functional automation $[6,9,10]$. Although water-quality monitoring technology has been researched in China at this decade, there are still many problems such as the unstable sensor performance, less real-time monitoring parameters of water quality, low level of networked monitoring, and single system network monitoring [11-13]. In recent years, many researches utilize new technology such as GPRS and neural networks to improve water quality monitoring level in China [14-15].

The pollution of aquaculture water, aquatic production and quality issues, and the establishment of food-safety traceability system make constructing effective dynamic monitoring network to obtain multi-parameters of water quality become an important issue in aquaculture. Distributed networking, real-time remote monitoring, the dynamic controlling of water quality using web-server-embedded technology and the sensor node technology are future trend development for intensive aquaculture [16]. In this study, a water-quality remote dynamic monitoring system was developed based on web-server-embedded technology. The information of water quality was transported safely using wireless communications technology based on CDMA services, WiFi and virtual private network (VPN) technology.

\section{Materials and Method}

\subsection{System Configuration}

The water-quality dynamic monitoring system is composed of water quality dynamic monitoring devices and a remote information server. The water quality dynamic monitoring device consists of sensor module, data monitoring module, communication module, and power module. The sensor module is composed of a water flow cell and sensors. The sensors include pH (ED201, Suzhou Han-star CO., China), electric conductivity (EC) (DJS-1T, Suzhou Han-star CO., China), dissolved oxygen (DO) (ED012, Suzhou Han-star CO., China), oxidation-reduction potential (ORP) (PC312, Suzhou Han-star CO., China), and temperature (STWB-1000, Beijing Saiyiling CO., China). The water flow cell was made of polyvinyl chloride (PVC) with diameter of $\Phi 55 \mathrm{~mm}$. The data monitoring module utilizes a web-server-embedded chip (PICNIC2.0, TriState Co., Japan) to obtain water-quality information and environment from different sensors. The communication module is composed of a Wi-Fi module (AirStation-J54, Buffalo Co., Japan), and a CDMA module with IPSec-based VPN function (InRouter210C, Beijing Inhand Co., China), for establishing wireless local area network and achieving remote communications and data transmission. The power module consists of a power control device, and a power division device for $12 \mathrm{~V}$ DC 
power supply and 220V AC power supply. The remote information server consists of an ADSL modem (DSL-300, D-Link Co., China), a VPN router (BV-601, NESCO Co., China), and an information server (Fig. 1). The VPN router and information server connect to the Internet via ADSL service. After the VPN router and the CDMA of the water-quality dynamic monitoring device are connected to the Internet, VPN connection is established via IPSec authentication. The information server is the terminal equipment which could be a personal computer, portable computer, or a large/medium size server to achieve the real-time remote capture of the water-quality parameter and environmental parameter of the aquiculture.

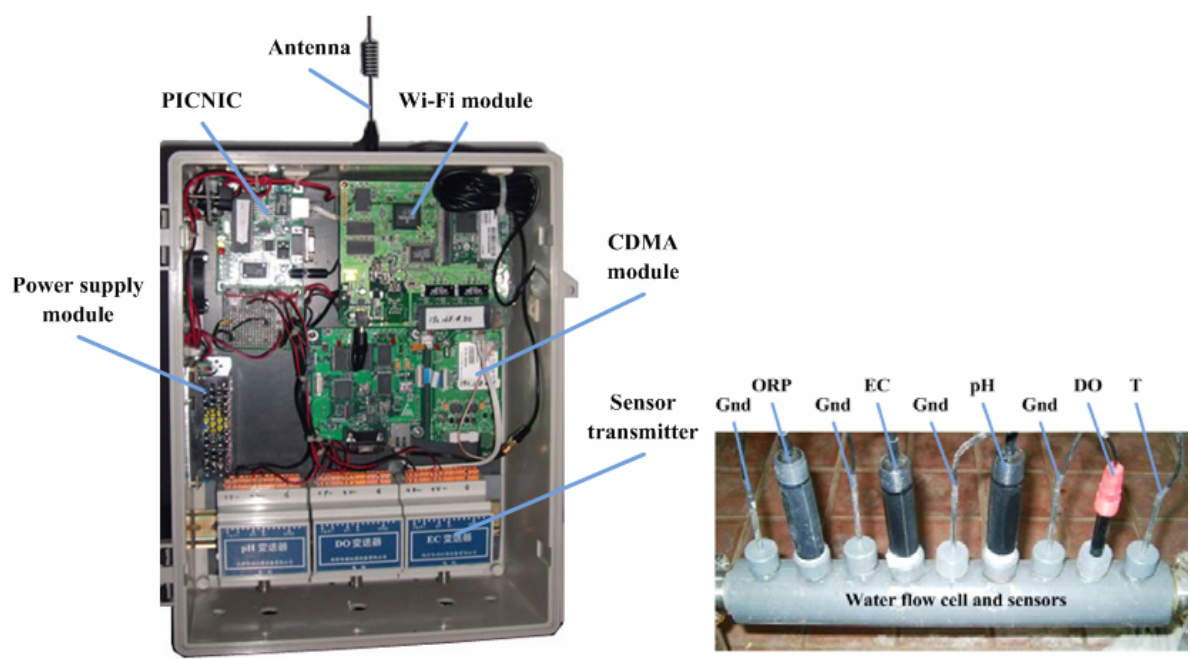

Fig. 1. The water-quality dynamic monitoring system

\subsection{Topological Architecture}

The water-quality dynamic monitoring system is an isolated local area network. As a sensing network node, it is connected to Internet via CDMA service of China Unicom. That means the system can be constructed to a large-scale wireless sensing network under CDMA signal covered areas. In this system, the two monitoring devices establish communication via Wi-Fi technology because of the short distance between them. The system is easy to increase by 255 monitoring devices at most, and the monitoring device is also easy to increase sensor channels. Water-quality data and environmental data are transported by TCP/IP protocol and identified and stored by the remote information server. The CDMA module will be dynamically connected to the remote information servers via IPSec-based VPN security technology. In order to identify the specified remote information servers, the remote VPN router have to use a dynamic domain or a fixed global IP to support the remote VPN calling. The monitoring systems 
deployed anywhere will become a local network connection if the IPsec-based VPN tunnels were connected. Within the network, the information captured by all devices could be used as a local information network to conduct secure access. Therefore, authorized users could visit or manage the remote sensing devices anywhere and anytime under Internet environment. In this testing, the Beijing information server is using a dynamic domain.

\subsection{Testing Environment}

Two water-quality dynamic monitoring systems were installed respectively in inlet and outlet of a workshop in Fengzeyuan aquafarm, Dongying Shandong Province, which adopts seawater and semi-circle mode for intensive aquiculture. Wi-Fi module was used in one of the devices, while Wi-Fi module and CDMA module with IPsec-based VPN function were used in another. In this testing, one remote information server with an IPsec-based VPN router is deployed in the China Agricultural University located in Beijing city. The testing experiments were conducted for two years.

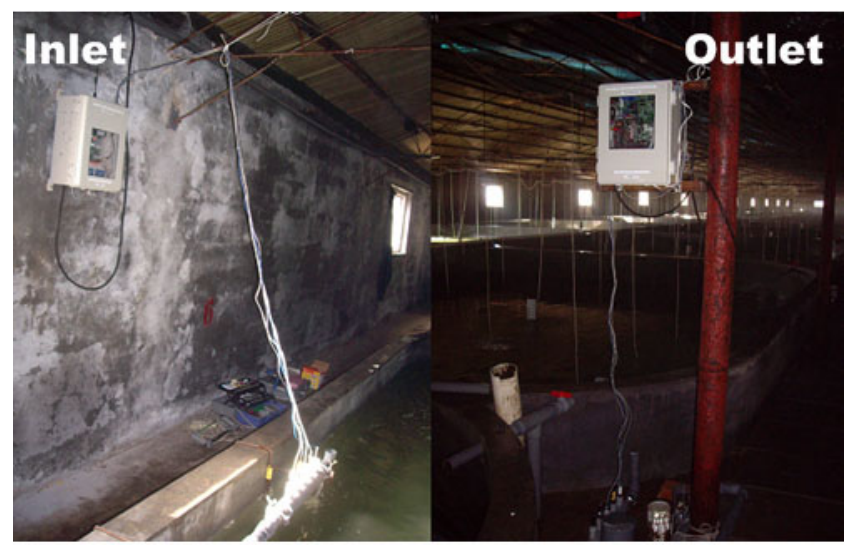

Fig. 2. The water-quality dynamic monitoring systems installed in a practical aquaculture

\section{Results and Discussion}

\subsection{Water-Quality Data Monitoring}

The water-quality data including $\mathrm{pH}, \mathrm{DO}, \mathrm{EC}$, and water temperature throughout a week were dynamically storied or issued in webpage or Extensible Markup Language (XML) file by a special JAVA applet program in the remote information server (Fig. 3). The 10-bit analog signals of the sensors were obtained by the web-server-embedded chip without storage device. 

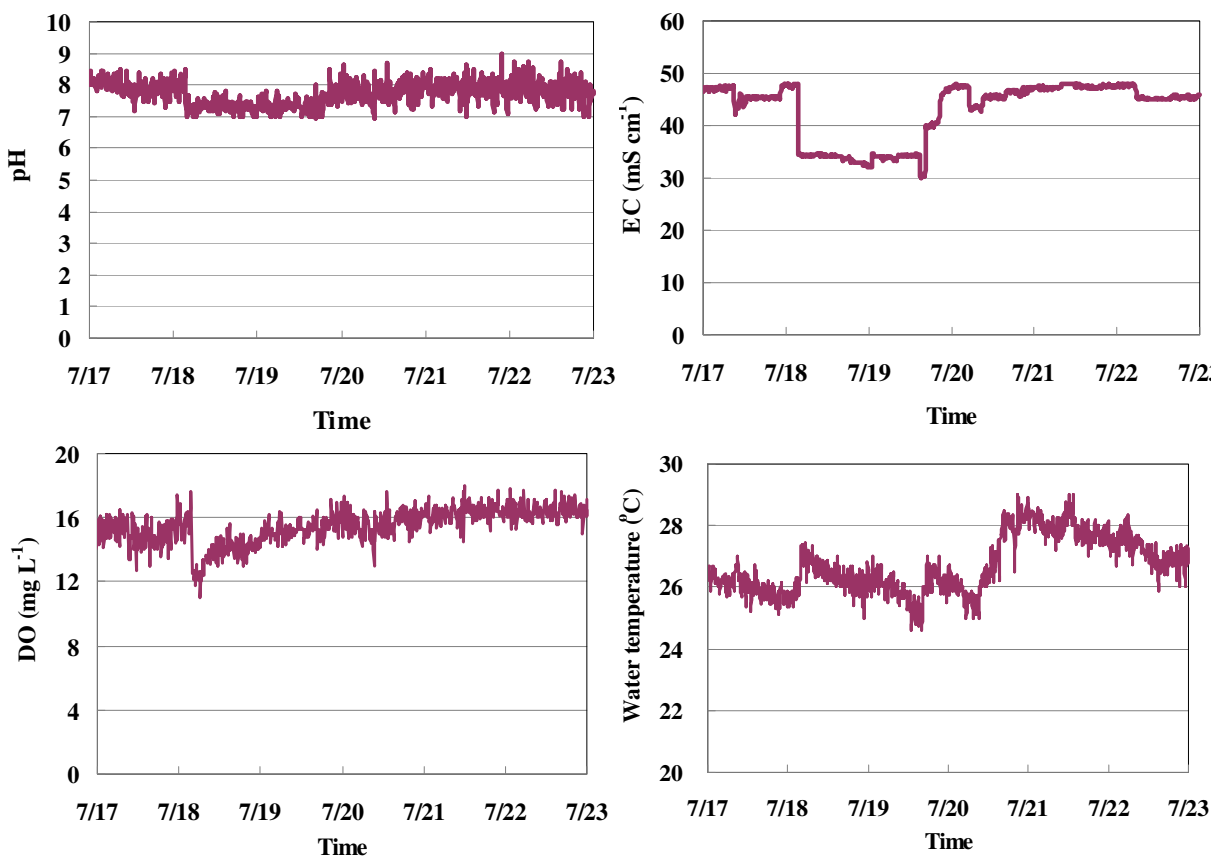

Fig. 3. Water-quality data measured by the water-quality dynamic monitoring system. These figures demonstrate the $\mathrm{pH}, \mathrm{EC}, \mathrm{DO}$ and water temperature data recorded within 6 days.

\subsection{Network Communication and Electronic Consumption}

The water-quality dynamic monitoring systems were communicated with the remote information server in Beijing by a 20-30Kbps access speed with over 26-30 signal quality level of CDMA services. One can access the CDMA device via LAN to inspect VPN status and network signal values. Also, other one can obtain real-time information of the system, including DNS and Public Network IP by viewing the logs. It is easy to fulfill local PC access to Internet by outside hanging a PC, setting IP address in the Subnet and DNS as the server detected by CDMA. Under normal processing of Internet, randomly select 6 days data to make research, we found that when setting the testing frequency for $1 \mathrm{~min}$ each time, that is 1440 sets per day, the average packet loss rate is between $1.7 \%$ to $2.3 \%$. Thus, this system has fulfilled real-time dynamic data acquisition and meets the needs in trust worthiness and safety of automatic network transmission.

The power controller of the water-quality dynamic monitoring system used $12 \mathrm{~V} \mathrm{DC}$ power supply with $2 \mathrm{~A}$ electric current. The nominal voltage of $\mathrm{pH}$, dissolved oxygen, electric conductivity, temperature and oxidation-reduction potential transmitters used $12 \mathrm{~V}$ DC power supply with $0.11 \mathrm{~A}$ measured electric current. The PICNIC and Wi-Fi need low consumption, the power of CDMA device is $4 \mathrm{~W}$ and the nominal voltage of fan is $12 \mathrm{~V}$. According to actual measurement of voltage and current on field devices, the average power is $12.9 \mathrm{~W}$. 


\section{Conclusion}

Information server of the water-quality dynamic monitoring system utilizes the value-added services of mobile telecommunication and the database management system based on web-server technology to conduct remote data transportation. The authorized users can obtain the monitoring water-quality data of the sensing network under any Internet environment. The results of two-year testing experiment shown that the water-quality dynamic monitoring system can remotely and dynamically monitor multi-parameter of water-quality such as temperature, $\mathrm{pH}, \mathrm{EC}, \mathrm{DO}$, and ORP. The system is operated well with $13 \mathrm{~W}$ power energy consumption. Therefore, this water-quality dynamic monitoring system can be used to construct a distributed water-quality monitoring network in multipoint of different cities for aquaculture farm and administration section in high reliability and security.

\section{Acknowledgments}

Thanks Mr. Po Yang and Mr. Chong Liu of Beijing Lighting Valley Company for their work and suggestions. Thanks Dongying Fengzeyuan aquafarm in Shandong province for supplying test site and their help. This research is supported by National advanced R\&D Program (2006BAD10A02 and 2006AA10A302).

\section{References}

1. China National Bureau of Statics: China Statistical Yearbook. China Statics Press, Beijing (2009) (in Chinese)

2. Charef, A., Ghauch, A., Baussand, P., Martin-Bouyer, M.: Water quality monitoring using a smart sensing system. Measurement 28, 219-224 (2000)

3. Park, S., Choi, J.H., Wang, S., Park, S.S.: Design of a water quality monitoring network in a large river system using the genetic algorithm. Ecol. Modell. 199, 289-297 (2006)

4. Strobl, R.O., Robillard, P.D.: Network design for water quality monitoring of surface freshwaters: A review. J. Environ. Manage. 87, 639-648 (2008)

5. Subasinghe, R.P.: Epidemiological approach to aquatic animal health management: opportunities and challenges for developing countries to increase aquatic production through aquaculture. Prev. Vet. Med. 67, 117-124 (2005)

6. Telci, I.T., Nam, K., Guan, J., Aral, M.M.: Optimal water quality monitoring network design for river systems. J. Environ. Manage. 90, 2987-2998 (2009)

7. Qi, Z., Zhang, X., Boon, N., Bossier, P.: Probiotics in aquaculture of China - Current state, problems and prospect. Aquaculture 290, 15-21 (2009)

8. Glasgow, H.B., Burkholder, J.M., Reed, R.E., Lewitus, A.J., Kleinman, J.E.: Real-time remote monitoring of water quality: a review of current applications, and advancements in sensor, telemetry, and computing technologies. J. Exp. Mar. Biol. Ecol. 300, 409-448 (2004)

9. Chilundo, M., Kelderman, P., Okeeffe, J.H.: Design of a water quality monitoring network for the Limpopo River Basin in Mozambique. Phys. Chem. Earth. 33, 655-665 (2008) 
10. Chen, L., Tan, C., Kao, S., Wang, T.: Improvement of remote monitoring on water quality in a subtropical reservoir by incorporating grammatical evolution with parallel genetic algorithms into satellite imagery. Water Res. 42, 296-306 (2008)

11. Zhu, M., Cao, G., Jiang, S., Han, S.: Monitor and control the parameter of the industrial aquaculture. Chin. J. Fish. 19, 99-104 (2006) (in Chinese)

12. Li, S.: Design of digital water quality parameters online monitoring system. Elect. Test. 160, 72-77 (2008) (in Chinese)

13. Liu, X., Sun, Y., Zhao, D., Cheng, L., Zhao, B., Qin, Y., Xu, W.: Research on intelligent supervising and control system about factors of aquiculture. Chin. J. Sci. Instrum. 27, 527-530 (2006) (in Chinese)

14. Cui, Y., Ban, J., Lu, W.: Research of distributed water-quality monitoring system based on network. Control Autom. 22, 167-169 (2006) (in Chinese)

15. Liu, X., Liu, L., Gao, J., Lu, J.: Research on integration of water quality automatic monitoring system with GPRS technology. J. Chin. Inst. of Water Resour. and Hydropower Res. 5, 75-79 (2007) (in Chinese)

16. Graveline, N., Maton, L., Rinaudo, J., Lukge, H., Interwies, E., Rouillard, J., Strosser, P., Palkaniete, K., Taverne, D.: An operational perspective on potential uses and constraints of emerging tools for monitoring water quality. Trends Analyt. Chem. 29, 378-384 (2010) 